\title{
Familial risks of aortic aneurysms among siblings in a nationwide Swedish study
}

Kari Hemminki, $M D, P h D^{1,2}$, Xinjun Li, $M D, P h D^{3}$, Sven-Erik Johansson, PhD ${ }^{3}$, Kristina Sundquist, $M D, P h D^{3}$, and Jan Sundquist, $M D, P h D^{3}$

\begin{abstract}
Purpose: Aortic aneurysms have a high fatality rate that could be reduced with control of risk factors and use of available screening methods for detection of early changes in aortic walls. The available data on familial risks, a potential indication for screening, are mainly limited to abdominal aortic aneurysms. Methods: A nationwide Swedish cohort was constructed by linking the Multigeneration Register on 0- to 69-year-old siblings to the Hospital Discharge Register and the Cause of Death Register for data on aortic aneurysms from years 1987 to 2001. Standardized incidence ratios (SIRs) were calculated for affected siblings by comparing with those whose siblings had no aneurysm. Results: A total of 71 affected siblings were identified with a familial SIR of 8.71; when one sibling was diagnosed before age 50 years, the SIR was 19.69. For concordant thoracic or concordant abdominal aneurysms, the SIRs were 21.68 and 13.06 , respectively. For brothers, the risk of abdominal aneurysms was 14.63, and 49.50 for diagnosis before age 50 years. Familial risks and the effects of early diagnostic age were shared by the anatomic subtypes of aneurysms. Within limits of the sample size, no gender differences could be observed. Affected siblings constituted $2.2 \%$ of all diagnosed patients. Conclusions: A family history of any aortic aneurysms and age groups younger than 50 years should be considered in recommendations for screening. The high familial risks are likely to be the result of heritable genes, the identification of which would allow gene testing and preventive counseling. Genet Med 2006:8(1):43-49.
\end{abstract}

Key Words: familial disease, sibling risk, heritability, screening, population-based

Aneurysms are swellings of the blood vessels, and on rupture, they are often fatal. Aneurysms are most common in the abdominal and thoracic parts of the aorta. The lesions in the ascending aorta are often dissections, that is, separations of aortic media that extend to distal segments of the affected vessel. $^{1-3}$ The known risk factors for aortic aneurysms include male gender, atherosclerosis, hypertension, smoking, vascular malformations, heritable traits, and family history. ${ }^{3-5}$ Atherosclerotic lesions preferentially affect the descending aorta, whereas medial degeneration is the most common lesion in the ascending aorta. ${ }^{2}$ Aneurysms are usually a result of a degenerative or an autoimmune disease, including, historically, latestage syphilitic infections. ${ }^{2,6,7}$ The pathogenesis of aneurysms involves reduction in elastic compliance of the aortic wall, reduction in medial matrix connective tissue fibers composed of fibrillin, elastin, and collagen, and proneness to progressive damage. ${ }^{2,3}$ Important mechanical components in this cascade are extracellular matrix defects, proteolytic enzymes, matrix

From the ${ }^{1}$ Division of Molecular Genetic Epidemiology, German Cancer Research Center (DKFZ), Heidelberg, Germany, ${ }^{2}$ Department of Biosciences at Novum, Karolinska Institute, Huddinge, and ${ }^{3}$ Center for Family Medicine, Karolinska Institute, Huddinge, Sweden.

Kari Hemminki, DKFZ, Im Neuenheimer Feld 580, D-69120 Heidelberg, Germany.

Received: July 25, 2005.

Accepted: October 31, 2005.

DOI: 10.1097/01.gim.0000195973.60136.48 metalloproteinases capable of degrading elastin and collagen, proteinase inhibitors, and persistent inflammation. ${ }^{3,5}$ Marfan syndrome is an autosomal dominant condition in which mutated fibrillin 1 prevents an orderly assembly of the elastic fibers. Dissections are one of the manifestations in this condition. ${ }^{2}$ Various types of vascular fragility are also found in excess in one form of Ehlers-Danlos syndrome, which is caused by defects in collagen structure and metabolism..$^{2,8}$

Familial clustering of aneurysms is well known, based on anecdotal reports and clinical series, covering both abdominal and thoracic aorta. Some $5 \%$ to $20 \%$ of the first-degree relatives of aneurysm patients have been reported to be affected.,3,9-11 Many of the genetic studies are old and small, and being patient series, they may have focused on selected populations. Probably for this reason the reported familial risks for abdominal aneurysms have varied widely from approximately 4 to more than 20 among first-degree relatives. ${ }^{3,11,12}$ Apparently the only population-based study, carried out in Utah using death certificates, found a much lower familial risk of 2.07 for abdominal aneurysms. ${ }^{13}$ Segregation analyses of families with abdominal aneurysms have shown complex patterns, with evidence for a recessive or dominant inheritance or no single inheritance pattern. ${ }^{14-16}$ Some obvious candidate genes underlying familial aggregation are the genes coding for matrix components, metalloproteinases, and their inhibitors and immune mediators, but, up to now, the tested candidates genes 
explain only a small proportion of the familial aggregation. ${ }^{3}$ Several putative candidate loci have been suggested in recent linkage studies, and mutations in transforming growth factor beta receptor type II have been detected in patients with thoracic aneurysms and dissections. ${ }^{17-20}$

In the present study we used the Swedish data on registered families and medically diagnosed aortic aneurysms, both with complete national coverage, to analyze familial risks between siblings in an unbiased setting. The Swedish family dataset, the Multigeneration Register, has been validated and extensively used in the study of familial cancer. ${ }^{21,22}$ Reliable data on familial risks for a potentially fatal disease, such as aneurysms, are of particular importance for clinical counseling and targeted surveillance. Such data benefit even gene identification strategies.

\section{MATERIALS AND METHODS}

The ad hoc research database used for this study, that is, the aneurysm database, was constructed by linking several national Swedish registers. Statistics Sweden, the Swedish government-owned statistics bureau, provided the Multigeneration Register in which persons (second generation) born in Sweden in 1932 and later are linked to their parents (first generation), registered shortly after birth. Families could be defined by linking all the children to their parents. Sibships can only be defined for the second generation, which was the present study population. Linkages were carried out using the unique personal identification number to the National Census Data (to obtain individual socioeconomic status) and to the Cause of Death Register. The final link was made by adding individual data from the Swedish Hospital Discharge Register that records complete data on all discharges with dates of hospitalization and diagnoses since 1986. All linkages were performed by the use of an individual national identification number that is assigned to each person in Sweden. This number was replaced by a serial number for each person to provide anonymity. The serial number was used to check that each individual was only entered once for his or her first appearance with an aneurysm diagnosis. More than 6.9 million individuals were included in the second generation of the aneurysm database.

\section{Outcome variable}

Data on patients diagnosed with aneurysms were retrieved from hospital discharges or cause of death notifications, reported according to the 9th (1987-1996) and 10th (19972001) version of the International Classification of Diseases (ICD). The subtypes of aneurysms included aortic dissection (no localization given), thoracic, thoracoabdominal, abdominal, and other aneurysms. Their ICD-9 codes were 441.0 dissection of aorta, 441.1-.2 thoracic aneurysms, 441.3-.4 abdominal aneurysms, 441.6-7 thoracoabdominal aneurysms, and 441.5 and 441.9 aortic aneurysms of unspecified site, defined as "others." According to the ICD-10, the grouping was identical, but the basic code was I71, instead of 441. A total of 3232 aneurysms were identified, 2656 from the Hospital Discharge Register and 576 (17.8\%) from the Cause of Death Register.

\section{Individual variables}

\section{Gender: Men and women}

Age at diagnosis was categorized as follows: less than 30 years, 30 to 39 years, 40 to 49 years, 50 to 59 years, and 60 to 69 years.

Socioeconomic status for the men and women was divided into six groups according to occupation: (1) farmers, (2) unskilled/ skilled workers, (3) white-collar workers, (4) professionals, (5) self-employed, and (6) all others.

Region was divided into three groups: ${ }^{1}$ large cities, ${ }^{2}$ southern Sweden, and ${ }^{3}$ northern Sweden.

Data on socioeconomic status and region were used as adjustment variables in the analysis, and no data on the distribution of these variables are given.

\section{Statistical analysis}

Person-years were calculated from start of follow-up on January 1, 1987, until the diagnosis of an aneurysm, death, emigration, or closing date, December 31, 2001. Age-specific incidence rates were calculated for the whole follow-up period, divided into five 5 -year periods. Standardized incidence ratios (SIRs) were calculated as the ratio of observed $(\mathrm{O})$ to expected (E) number of cases. The expected number of cases was calculated for age (5-year groups), sex, period (5-year groups), region, and socioeconomic status-specific standard incidence rates. Sibling risks were calculated for men and women with siblings affected with aneurysms, compared with men and women whose siblings were not affected by aneurysms, using the cohort methods as described. ${ }^{23}$ Confidence intervals (95\% CI) were calculated assuming a Poisson distribution and adjusted for dependence between the sibling pairs. ${ }^{23}$ Age-adjusted incidence rates were calculated on the basis of the European standard population. The population attributable fraction (PAF) of cases with a family history of aneurysm was estimated as follows: proportion of familial cases $\times$ (familial SIR-1)/familial SIR. ${ }^{24}$

\section{RESULTS}

A total of 3232 cases of aneurysm were diagnosed in the 0 - to 69-year-old second generation in the Swedish aneurysm database between years 1987 and 2001 (Table 1). Abdominal aneurysms accounted for $39.5 \%$ of all aneurysms, followed by aortic dissections with no localization given (30.4\%), thoracic (19.9\%), and thoracoabdominal (3.6\%) aneurysms. For the group "others" (6.7\%), the location was not defined. The agespecific incidence rates for the different types are shown in Figure 1, separately for men and women (note the 10-fold difference in scale). There was an age-dependence for all aneurysm types. The incidence of abdominal aneurysms was approximately 10 times higher for men than women; the gender difference was approximately threefold for dissections, which 
Table 1

Number of cases of aneurysms in the 0 - to 69 - year- old study population

\begin{tabular}{|c|c|c|c|c|c|c|c|c|c|c|c|c|}
\hline \multirow[b]{3}{*}{ Age at diagnosis (y) } & \multicolumn{12}{|c|}{ Subtype of aneurysm } \\
\hline & \multicolumn{2}{|c|}{ Dissection } & \multicolumn{2}{|c|}{ Thoracic } & \multicolumn{2}{|c|}{ Abdominal } & \multicolumn{2}{|c|}{ Thoracoabdominal } & \multicolumn{2}{|c|}{ Others } & \multicolumn{2}{|c|}{ All } \\
\hline & No. & $\%$ & No. & $\%$ & No. & $\%$ & No. & $\%$ & No. & $\%$ & No. & $\%$ \\
\hline$<30$ & 50 & 5.1 & 68 & 10.6 & 4 & 0.3 & 4 & 3.5 & 13 & 6.0 & 139 & 4.3 \\
\hline $30-39$ & 70 & 7.1 & 79 & 12.3 & 8 & 0.6 & 9 & 7.8 & 10 & 4.7 & 176 & 5.4 \\
\hline $40-49$ & 232 & 23.6 & 106 & 16.5 & 53 & 4.2 & 13 & 11.3 & 34 & 15.8 & 438 & 13.6 \\
\hline $50-59$ & 406 & 41.4 & 252 & 39.1 & 516 & 40.4 & 54 & 47.0 & 80 & 37.2 & 1308 & 40.5 \\
\hline $60-69$ & 223 & 22.7 & 139 & 21.6 & 696 & 54.5 & 35 & 30.4 & 78 & 36.3 & 1171 & 36.2 \\
\hline All & 981 & 100.0 & 644 & 100.0 & 1277 & 100.0 & 115 & 100.0 & 215 & 100.0 & 3232 & 100.0 \\
\hline
\end{tabular}
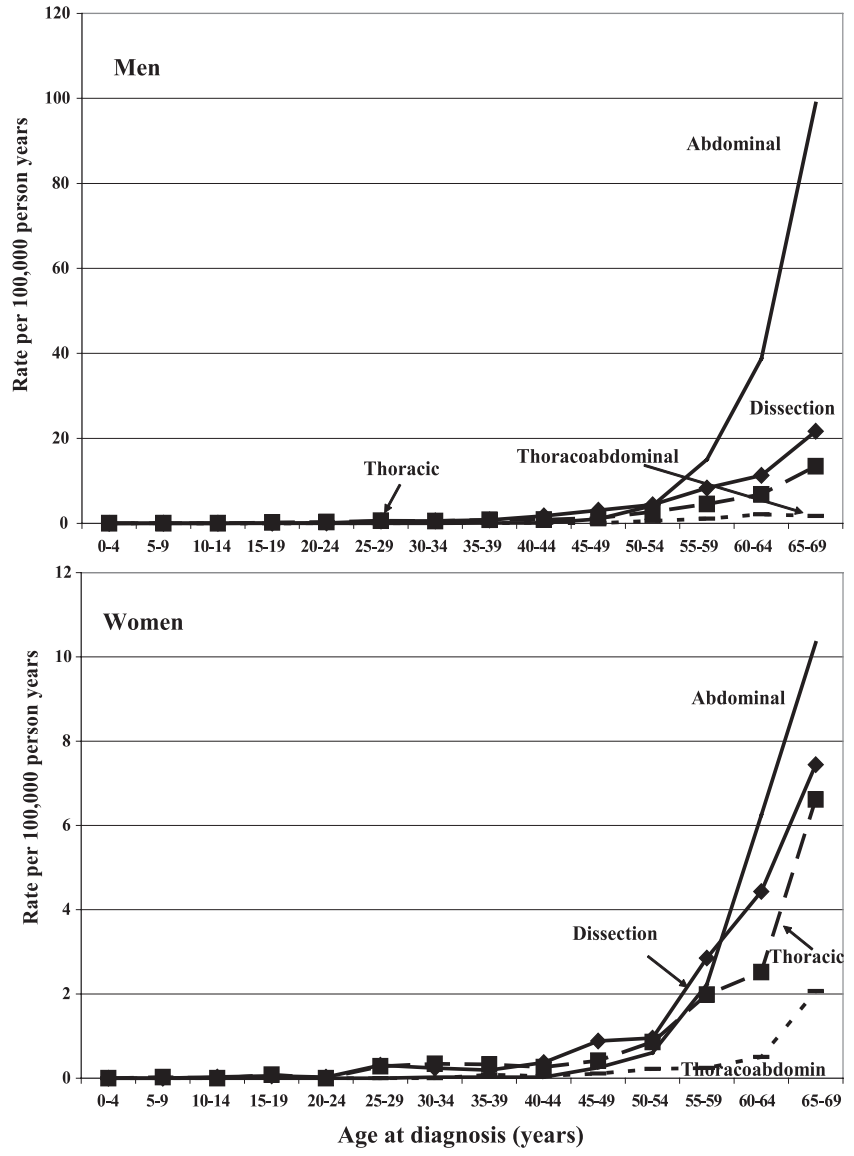

Fig. 1 Age-specific incidence rates for different aneurysm subtypes.

were relatively common at a younger age, and twofold for thoracic aneurysms.

Figure 2 shows the age-specific incidence rates of all aneurysms among siblings with or without a sibling history of aneurysms. The familial incidence rate showed early- and lateonset components with high incidence, separated by a low incidence in the age group 40 to 44 years. All of the female cases aged less than 50 years (five in total) occurred in the age group 30 to 34 years, in which the incidence was equal to the male familial incidence.
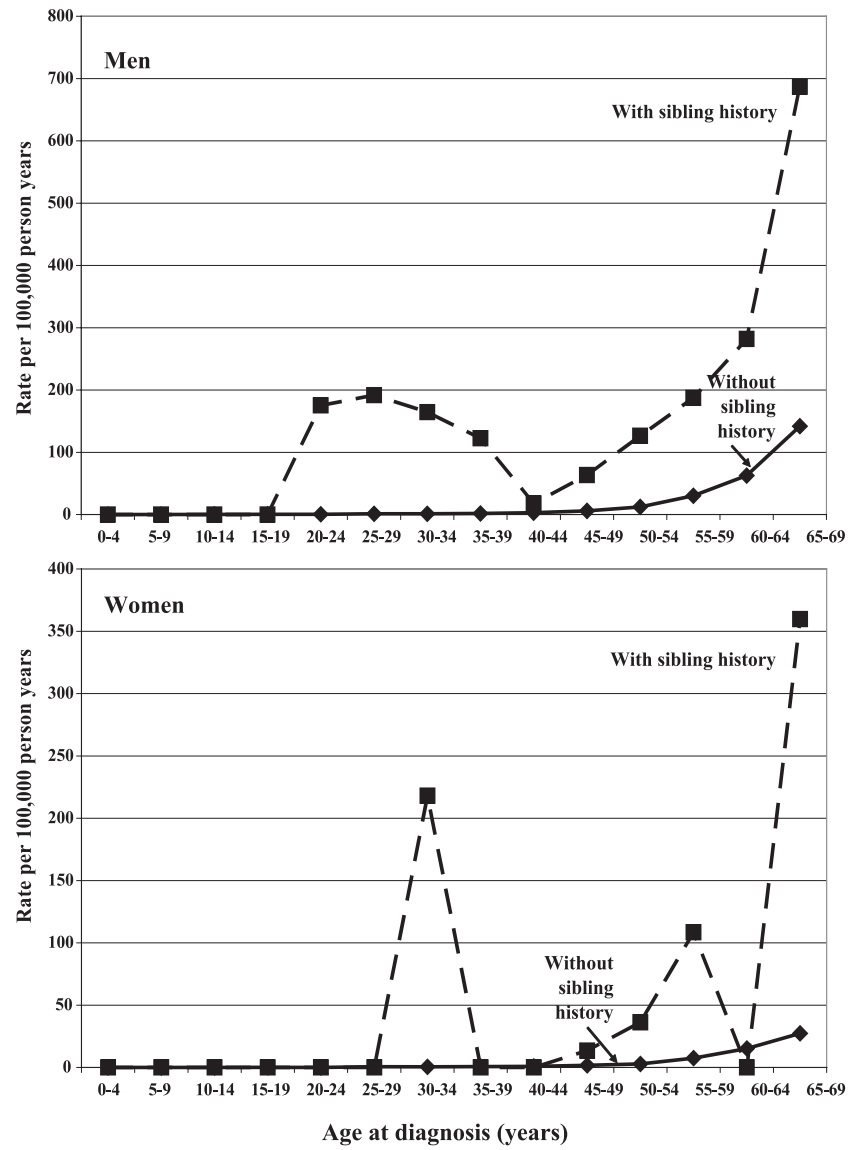

Fig. 2 Age-specific incidence rates for familial (with sibling history) and sporadic (without sibling history) aneurysms. The difference between the two curves gives an approximation for the familial risk.

Table 2 shows age-specific SIRs of aneurysms among siblings according to the aneurysm subtypes. The overall risks were highest for thoracoabdominal aneurysms, followed by thoracic and abdominal aneurysms and dissections. Diagnosis of at least one sibling before age 50 years was an important risk factor for all types of aneurysms. In this group, the SIRs for thoracoabdominal and thoracic aneurysms were remarkably high, 64.79 and 40.05. For thoracic and abdominal aneurysms 


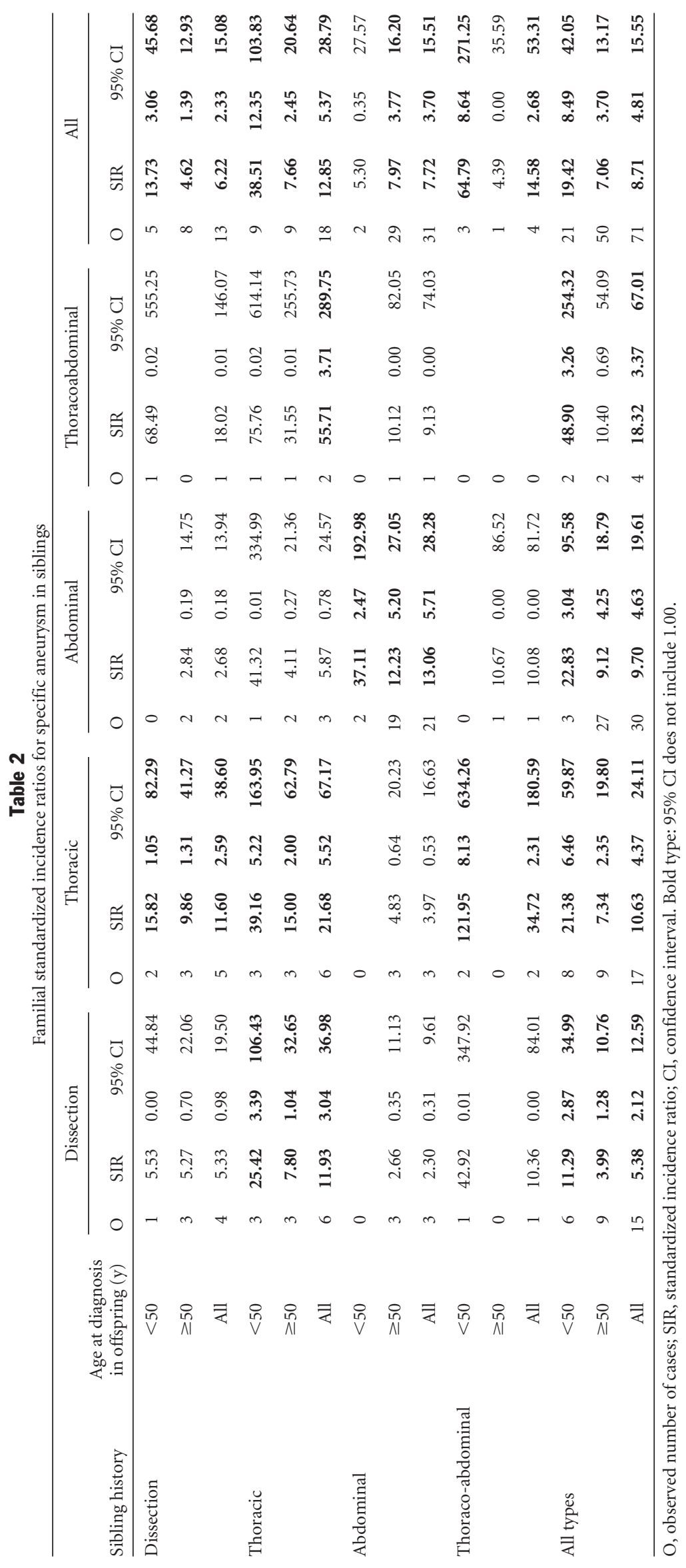


Table 3

Familial standardized incidence ratios for abdominal aneurysm in male and female siblings

\begin{tabular}{|c|c|c|c|c|c|c|c|c|c|c|c|c|c|}
\hline \multirow{3}{*}{$\frac{\text { Gender of sibling }}{\text { Men }}$} & \multirow{3}{*}{$\begin{array}{l}\begin{array}{c}\text { Age at diagnosis } \\
\text { in offspring }(y)\end{array} \\
<50\end{array}$} & \multicolumn{4}{|c|}{ Men } & \multicolumn{4}{|c|}{ Women } & \multicolumn{4}{|c|}{ All } \\
\hline & & \multirow{2}{*}{$\frac{\mathrm{O}}{2}$} & \multirow{2}{*}{$\frac{\text { SIR }}{49.50}$} & \multicolumn{2}{|c|}{$95 \%$ CI } & \multirow{2}{*}{$\frac{\mathrm{O}}{0}$} & \multirow[t]{2}{*}{ SIR } & \multicolumn{2}{|c|}{$95 \%$ CI } & \multirow{2}{*}{$\frac{\mathrm{O}}{2}$} & \multirow{2}{*}{$\frac{\text { SIR }}{43.29}$} & \multicolumn{2}{|c|}{$95 \%$ CI } \\
\hline & & & & 3.30 & 257.47 & & & & & & & 2.89 & 225.15 \\
\hline & $\geq 50$ & 15 & 13.37 & 5.27 & 31.26 & 2 & 9.76 & 0.65 & 50.77 & 17 & 12.81 & 5.26 & 29.07 \\
\hline & All & 17 & 14.63 & 6.01 & 33.19 & 2 & 9.49 & 0.63 & 49.37 & 19 & 13.84 & 5.88 & 30.62 \\
\hline \multirow[t]{3}{*}{ Women } & $<50$ & 0 & & & & 0 & & & & 0 & & & \\
\hline & $\geq 50$ & 2 & 9.91 & 0.66 & 51.55 & 0 & & & & 2 & 8.81 & 0.59 & 45.82 \\
\hline & All & 2 & 9.59 & 0.64 & 49.89 & 0 & & & & 2 & 8.52 & 0.57 & 44.30 \\
\hline \multirow[t]{3}{*}{ All } & $<50$ & 2 & 42.55 & 2.84 & 221.32 & 0 & & & & 2 & 37.11 & 2.47 & 192.98 \\
\hline & $\geq 50$ & 17 & 12.84 & 5.28 & 29.14 & 2 & 8.69 & 0.58 & 45.21 & 19 & 12.23 & 5.20 & 27.05 \\
\hline & All & 19 & 13.86 & 5.89 & 30.67 & 2 & 8.44 & 0.56 & 43.89 & 21 & 13.06 & 5.71 & 28.28 \\
\hline
\end{tabular}

O, observed number of cases; SIR, standardized incidence ratio; CI, confidence interval. Bold type: 95\% CI does not include 1.00 .

the risks seemed to be highest for concordant (both siblings same type) compared with discordant aneurysm types. The risk for all aortic aneurysms was marginally higher for women, $9.93(\mathrm{~N}=18,95 \%$ CI $4.15-22.24)$, than for men, $8.36(\mathrm{~N}=53$, 95\% CI 4.43-15.48). If one sibling was diagnosed before age 50 years, the familial SIR was marginally higher for men, 20.57 $(\mathrm{N}=16,95 \% \mathrm{CI} 8.29-47.35)$, than for women, $17.33(\mathrm{~N}=5$, 95\% CI 3.87-57.65).

In Table 3, gender-specific risks of abdominal aneurysms were considered. In brother pairs, the overall SIR was 14.63 and the risk was higher, 49.50 when one brother was diagnosed before age 50 years. No concordant sister pairs were found, but the risk for the two sisters with affected brothers was 9.49 (95\% CI 0.63-49.37).

In this 0 - to 69 -year-old population, 71 familial aneurysms accounted for $2.2 \%$ (familial proportion) of all 3232 aneurysms, giving a familial PAF of $1.9 \%$.

\section{DISCUSSION}

The mortality and morbidity from aortic aneurysms are high, but efficient screening methods are available, primarily using ultrasonography. ${ }^{25,26}$ An unambiguous understanding of the risk factors is required for an optimal use of the screening possibilities for prevention. However, regarding the family history no such uniform acceptance seems to exist. The recent screening recommendations for abdominal aneurysms differ on the indication of family history; one recommends screening of men and women older than 50 years with a positive family history, ${ }^{25}$ and the other recommends primarily men with a first-degree family history of an abdominal aortic aneurysm requiring surgical repair. ${ }^{26}$ Why do the available data not allow a more uniform assessment? A recent review on the pathobiology of aortic aneurysms in the section "Genetics" discussed approximately 20 articles relating to the topic of familial risk. ${ }^{3}$ All of these were clinical series, published between the late 1970s and early 1990s. The contrast in the risk estimates from these early publications to the only available population-based study was noted in the latter study, whereas the risks to firstdegree relatives in the early studies were cited to range from 11 to 18. The authors' own population-based results showed a twofold risk; however, the authors assumed that their own results were an underestimate. ${ }^{13}$ It seems obvious that the currently available familial risk estimates for aortic aneurysms are too variable to be used as a basis for clinical recommendations. Furthermore, they are practically only limited to abdominal aneurysms. The problems with selection and reporting biases in familial risk estimates from clinical series and even from case-control studies are well known in cancer studies, in which the present Multigeneration Register has been instrumental in providing reliable risk estimates. ${ }^{27}$ However, we need to admit that there are also limitations to the available data, as discussed next.

The Cause of Death Register (17.8\% of aneurysms) and the Hospital Discharge Register ( $82.2 \%$ of aneurysms) were the sources of present diagnostic data used to construct the aneurysm database. A study assessing diagnostic accuracy in Swedish medical statistics from the year 1995 found a good concordance between hospital discharge diagnoses and the underlying causes of death for "dramatic conditions," such as diseases of arteries, arterioles, and capillaries, with an $89 \%$ agreement. ${ }^{28}$ We can thus assume that the overall level of diagnostic data is good. Acute fatal conditions, such as rupturing aortic aneurysm, may be misdiagnosed as an acute myocardial infarct. ${ }^{29}$ Such misdiagnosis would decrease true familial risks. We believe that this may not be a large problem in the present study because only a small proportion of subjects were identified from the Cause of Death Register and because autopsy rates have been high in Sweden, particularly when the diagnosis has been in doubt, as in acute deaths. However, the present analysis has some important limitations. Because the present data spanned only 15 years (1987-2001) we could consider only one generation, siblings, and the number of affected siblings was no more than 71 . If the inheritance patterns were 
recessive, the present familial results could be higher than would be expected for parent-offspring pairs; however, the available literature, given its limitations, lends no strong support for a recessive mechanism. ${ }^{3,13}$

The overall familial risk of 8.71 observed in the present study was very high. However, only $2.2 \%$ of all aneurysms in this 0 - to 69-year-old population were familial, amounting to a PAF of $1.9 \%$. The PAF is a function of the familial risk and proportion, both of which are dependent on the age of the population. Of course, the PAF depends on the definition of the familial relationship, all first-degree relatives, parent-offspring, or, as used here, siblings. The familial risk for concordant aneurysms was high for thoracic (21.68) and abdominal (13.06) aneurysms; for both types, the SIRs were two to three times higher when at least one sibling was diagnosed before age 50 years. Marfan syndrome could have contributed to the early-onset cases (Fig. 2), but it was not likely to be the only reason because less than half of the early-onset cases were dissections and thoracic aneurysms, the most common sites in this syndrome; unfortunately no further details were available. The overall familial risks were highest for thoracoabdominal aneurysms and lowest for dissections, but concordant subtypes for these aneurysms could not be assessed because there were too few affected sibling pairs. With the limits of the present sample size, the overall risks to men and women were equally high, even though the incidence of aneurysms was much higher among men. The implication is that the familial risk is proportional to the background incidence. For abdominal aneurysms, with a high male incidence, SIRs for concordant aneurysms could be assessed specifically for men, being 14.63 for all men and 49.50 when one brother was diagnosed before age 50 years. No concordant sister pairs with abdominal aneurysms were found. However, on the basis of the two detected sister-brother pairs, the risk to women could not be excluded.

In regard to the screening recommendations for abdominal aneurysms, the present data have some relevant implications. ${ }^{25,26}$ First, the consideration of the family history should not be limited to abdominal aneurysms, because all aortic aneurysms seem to share the familial risk factors. Yet, the present data lend some support to the higher concordant risks for abdominal aneurysms compared with discordant types. Second, to screen individuals with a family history only after age 50 years will miss high-risk individuals. Admittedly, earlyonset familial cases are rare for abdominal aneurysms, but they are more common for other aortic aneurysms. In the present series 2 of $21(9.5 \%)$ of familial abdominal aneurysms were diagnosed before age 50 years, compared with 21 of 71 (29.6\%) for all aortic aneurysms. Very little is known about international incidence levels for aortic aneurysms, and we have thus no possibility to assess the generalizability of the present findings. ${ }^{29,30}$

In summary, the present data on medically verified cases of aneurysms from registered family structures covering all of Sweden showed high familial risks between siblings for aortic aneurysms. The risks were shared by the anatomic subtypes, and no gender differences were observed, within limits of the present sample size. Diagnosis before age 50 years carried a twofold to threefold risk compared with diagnosis at a higher age. A family history of any aortic aneurysms should be considered in recommendations for screening, and these should be extended to age groups younger than 50 years. The high familial risks are likely to be the result of heritable genes, the identification of which would allow gene testing and preventive counseling and screening, directed in the most cost-efficient manner to those at higher risk.

\section{ACKNOWLEDGMENTS}

This work was supported by grants from the National Institutes of Health, the Swedish Council for Working Life and Social Research, and the Swedish Research Council. The registers used in the present study are maintained at Statistics Sweden and the National Board of Health and Welfare.

\section{References}

1. Kumar V, Cotran R, Robbins S. Basic Pathology. Philadelphia: WB Saunders; 1997.

2. Hasham SN, Guo DC, Milewicz DM. Genetic basis of thoracic aortic aneurysms and dissections. Curr Opin Cardiol 2002;17:677-683.

3. Alexander J. The pathology of aortic aneurysms. J Surg Res 2004;117:163-175.

4. Verloes A, Sakalihasan N, Limet R, Koulischer L. Genetic aspects of abdominal aortic aneurysm. Ann N Y Acad Sci 1996;800:44-55.

5. van Vlijmen-van Keulen CJ, Pals G, Rauwerda JA. Familial abdominal aortic aneurysm: a systematic review of a genetic background. Eur J Vasc Endovasc Surg 2002; 24:105-116.

6. Hirose H, Takagi M, Miyagawa N, Hashiyada H et al. Genetic risk factor for abdominal aortic aneurysm: HLA-DR2(15), a Japanese study. J Vasc Surg 1998;27:500-503.

7. Hirose H, Tilson MD. Abdominal aortic aneurysm as an autoimmune disease. Ann N Y Acad Sci 2001;947:416-418.

8. Myllyharju J, Kivirikko KI. Collagens, modifying enzymes and their mutations in humans, flies and worms. Trends Genet 2004;20:33-43.

9. Biddinger A, Rocklin M, Coselli J, Milewicz DM. Familial thoracic aortic dilatations and dissections: a case control study. J Vasc Surg 1997;25:506-511.

10. Coady MA, Davies RR, Roberts M, Goldstein LJ et al. Familial patterns of thoracic aortic aneurysms. Arch Surg 1999;134:361-367.

11. Salo JA, Soisalon-Soininen S, Bondestam S, Mattila PS. Familial occurrence of abdominal aortic aneurysm. Ann Intern Med 1999;130:637-642.

12. Webster MW, St Jean PL, Steed DL, Ferrell RE et al. Abdominal aortic aneurysm: results of a family study. J Vasc Surg 1991;13:366-372.

13. Cannon Albright LA, Camp NJ, Farnham JM, MacDonald J et al. A genealogical assessment of heritable predisposition to aneurysms. J Neurosurg 2003;99:637-643.

14. Majumder PP, St Jean PL, Ferrell RE, Webster MW et al. On the inheritance of abdominal aortic aneurysm. Am J Hum Genet 1991;48:164-170.

15. Verloes A, Sakalihasan N, Koulischer L, Limet R. Aneurysms of the abdominal aorta: familial and genetic aspects in three hundred thirteen pedigrees. J Vasc Surg 1995; 21:646-655.

16. Kuivaniemi H, Shibamura H, Arthur C, Berguer R et al. Familial abdominal aortic aneurysms: collection of 233 multiplex families. J Vasc Surg 2003;37:340-345.

17. Kakko S, Raisanen T, Tamminen M, Airaksinen J et al. Candidate locus analysis of familial ascending aortic aneurysms and dissections confirms the linkage to the chromosome 5q13-14 in Finnish families. J Thorac Cardiovasc Surg 2003;126:106-113.

18. Hasham SN, Willing MC, Guo DC, Muilenburg A et al. Mapping a locus for familial thoracic aortic aneurysms and dissections (TAAD2) to 3p24-25. Circulation 2003; 107:3184-3190.

19. Shibamura H, Olson JM, van Vlijmen-Van Keulen C, Buxbaum SG et al. Genome scan for familial abdominal aortic aneurysm using sex and family history as covariates suggests genetic heterogeneity and identifies linkage to chromosome 19q13. Circulation 2004;109:2103-2108.

20. Pannu H, Fadulu VT, Chang J, Lafont A et al. Mutations in transforming growth factor-beta receptor type II cause familial thoracic aortic aneurysms and dissections. Circulation 2005;112:513-520.

21. Hemminki K, Li X. Familial risks of cancer as a guide to gene identification and mode of inheritance. Int J Cancer 2004;110:291-294. 
22. Hemminki K, Li X, Czene K. Familial risk of cancer: data for clinical counseling and cancer genetics. Int J Cancer 2004;108:109-114.

23. Hemminki K, Vaittinen P, Dong C, Easton D. Sibling risks in cancer: clues to recessive or X-linked genes? Br J Cancer 2001;84:388-391.

24. Miettinen O. Proportion of disease caused or prevented by a given exposure, trait or intervention. Am J Epidemiol 1974;99:325-332.

25. Kent KC, Zwolak RM, Jaff MR, Hollenbeck ST et al. Screening for abdominal aortic aneurysm: a consensus statement. J Vasc Surg 2004;39:267-269.

26. US Preventive Services Task Force. Screening for abdominal aortic aneurysms: recommendation statement. Ann Intern Med 2005;142:198-202.
27. Hemminki K, Eng C. Clinical genetic counselling for familial cancers requires reliable data on familial cancer risks and general action plans. J Med Genet 2004;41:801-807.

28. Johansson LA, Westerling R. Comparing Swedish hospital discharge records with death certificates: implications for mortality statistics. Int J Epidemiol 2000;29:495502.

29. Hagan PG, Nienaber CA, Isselbacher EM, Bruckman D et al. The International Registry of Acute Aortic Dissection (IRAD): new insights into an old disease. JAMA 2000;283:897-903.

30. Nienaber CA, Fattori R, Mehta RH, Richartz BM et al. Gender-related differences in acute aortic dissection. Circulation 2004;109:3014-3021. 